\title{
THE KINETICS OF THE $\gamma$ ' PHASE AND ITS STRAIN IN THE NICKEL BASE SUPERALLOY SC16 STUDIED BY IN-SITU NEUTRON AND SYNCHROTRON RADIATION DIFFRACTION.
}

\author{
G.Bruno ${ }^{1}$, H.Cavalcanti Pinto ${ }^{2}$ \\ ${ }^{1}$ Institut Laue Langevin, Diffraction Group, BP 156, F-38042 Grenoble, France \\ ${ }^{2}$ Institute for Materials Science and Testing, Vienna University of Technology, Karlsplatz 13, A-1040 Vienna, Austria
}

Keywords: SC16, single crystals, misfit, coherency strains, nucleation and growth, high resolution diffraction

\begin{abstract}
In-situ studies of the kinetics of the nucleation and growth of the $\gamma^{\prime}$ phase, as well as of the phase strains in the single crystal Nibase superalloy SC16 were carried out by means of neutron and high-energy synchrotron radiation diffraction. The investigations were aimed at a better understanding of the fundamental kinetic phenomena, in view of the optimization of the heat treatments applied prior to the industrial use of the alloy, and at monitoring the evolution/relaxation of the interphase strains during the microstructural changes.

Measuring superlattice reflections allows the different stages of decomposition, nucleation, growth and concurrent coarsening to be followed by in-situ neutron diffraction. Results show that at temperatures $\mathrm{T} \leq 1490 \mathrm{~K}$ (a critical undercooling of about $30 \mathrm{~K}$ is found) an increasing volume fraction of $\gamma^{\prime}$ can be found with decreasing temperature, until a saturation at around $1100 \mathrm{~K}$ occurs. Following classical kinetics theory, an Avrami-like growth (time) law was used to model the volume fraction time behaviour.

The total reaction rate $\mathrm{W}$ shows a maximum at around $1300 \mathrm{~K}$, where the competing nucleation and growth processes find equilibrium. The reaction rate first increases with the undercooling $\Delta \mathrm{T}$ and then decreases. This implies a change in the character of the reaction, which was found to be initially surface and then bulk driven.

Unlike the integrated intensity, the lattice mismatch follows a pure exponential time decay, having bigger decay times at higher temperatures. This implies that the particles are born fully coherent, and then coherency strains set up. As the time goes by, long term ageing shows a stabilisation of the misfit value, while the precipitates are supposed to loose their coherency to the matrix (already within 3-4 h), and the strains relax.

The strain relaxation rate has its maximum around $1420 \mathrm{~K}$.
\end{abstract}

\section{Introduction}

The development from polycrystalline to single crystal turbine parts has led to an improvement of the mechanical properties and an increase of the operating temperature of Nickel base superalloys. The superalloy SC16 belongs to the first generation of single crystal materials for stationary turbines and to the low $\gamma^{\prime}$ volume fraction (about 40\%) alloy class. Its composition is given in Table 1. Although more advanced alloys have been developed, which contain heavy refractory elements, the low number of compositional elements in SC16 gives the advantage of an easier modeling of the diffusion properties.

The exceptional performance of single crystal superalloys comes from the particular arrangement of the precipitate phase, $\gamma$, which is isostructural with the fcc $\gamma$ matrix in which it is embedded coherently, but is ordered $\left(\mathrm{Ni}_{3}(\mathrm{Al}, \mathrm{Ti})\right)$ with a $\mathrm{Ll}_{2}$ structure. A very high shear resistance at high temperature, along with an increase of the flow stress around 1000-1100 K [1,2], renders these alloys particularly attractive. While cooling the alloy SC16, after being homogenized at $1525 \mathrm{~K}$ (usually for $3 \mathrm{~h}$ ), the $\gamma^{\prime}$ phase builds up at around $1425 \mathrm{~K}$, but its final form (usually cuboidal) and size (usually around $400 \mathrm{~nm}$ edge) are only achieved after an ageing treatment for $24 \mathrm{~h}$ at $1125 \mathrm{~K}$. This is called the 'standard heat treatment', SHT, for this alloy [3]. There are three driving mechanisms which rule the kinetics of the $\gamma^{\prime}$ phase [4]: the interfacial energy, $\sigma_{\gamma \gamma}$, the coherency energy, $E_{\delta}$, and the Helmoltz energy of $\gamma^{\prime}$ nucleation, $F_{\gamma^{\prime}}$. For a (spherical) particle of radius $\mathrm{R}$ they combine as

$\Delta F(R)=\frac{4}{3} \pi R^{3}\left(-\Delta F_{\gamma^{\prime}}+\Delta E_{\delta}\right)+4 \pi R^{2} \sigma_{\gamma \gamma^{\prime}}$

(where the $\gamma^{\prime}$ forms only if the total Helmoltz energy $\Delta F<0$ ).

The first two contributions have a temperature dependence and their relative weight brings the reaction to be 'surface' or 'strain' driven: the prevalence of one of the terms changes drastically the shape and the size of the precipitates.

If we define the $\gamma^{\prime}-\gamma$ lattice misfit $\delta$, as

$\delta=\frac{a_{\gamma^{\prime}}-a_{\gamma}}{\frac{1}{2}\left(a_{\gamma^{\prime}}+a_{\gamma}\right)}$

with $a_{\gamma}, a_{\gamma^{\prime}}$ as the lattice parameters of the $\gamma$ and $\gamma^{\prime}$ phase, respectively, this quantity will be proportional to the coherency strains between the two phases. The coherency strain strongly influences the creep behavior of the alloy $[5,6]$. Concurrently, the amount of diffusion alters the volume fraction, the size and the form of the $\gamma^{\prime}$ particles, so that both energy terms $E_{\delta}$ and $F_{\gamma}$, are very important for the evolution of microstructure and mechanical properties. Upon cooling to just below the solvus temperature $\left(\mathrm{T}_{0}\right)$ of the $\gamma^{\prime}$ phase, one can find a temperature where $\gamma^{\prime}$ starts forming, depending on the $\mathrm{Al}$ and $\mathrm{Ti}$ concentrations. If we cool further, and age for a sufficiently long time, a growth and competitive coarsening of the $\gamma^{\prime}$ particles is expected to occur, but still nucleation of new small particles will take place at the beginning of each treatment.

Some investigations have already been performed by TEM $[3,7,9]$ on quenched samples and others were conducted starting from the SHT condition and then heating up to $1470 \mathrm{~K}[10,11]$, using synchrotron radiation diffraction. However, under such conditions, no nucleation of new $\gamma^{\prime}$-precipitates occurred. In the present work the experimental difficulty of working in the small 
temperature window between solvus and melting point could be overcome.

Table 1. Composition of the SC16 superalloy and scattering lengths of its constituents for thermal neutrons.

\begin{tabular}{|l|l|l|l|l|l|l|}
\hline Element & $\mathrm{Ni}$ & $\mathrm{Al}$ & $\mathrm{Ti}$ & $\mathrm{Ta}$ & $\mathrm{Cr}$ & $\mathrm{Mo}$ \\
\hline \%at & 68.1 & 7.35 & 4.15 & 1.1 & 17.5 & 1.8 \\
$\mathrm{~b}\left(10^{-14} \mathrm{~m}\right)$ & 1.03 & 0.34 & -0.34 & 0.69 & 0.36 & 0.70 \\
\hline
\end{tabular}

The scope of this work was to focus on the early stages of the $\gamma^{\prime}$ nucleation, where the volume fraction belonging to a particular ageing temperature is still a function of time. Real-time neutron and synchrotron radiation diffraction were used. Neutron and synchrotron radiation diffraction have been very advantageously employed for in situ real-time studies [10,11], because of the reduced counting times with respect to conventional $\mathrm{x}$-ray sources [12] and the versatility of the sample environment (this can be at least problematic for other standard techniques).

Moreover, the intensity and resolution achievable at a synchrotron beamline are at least one order of magnitude higher than the most advanced laboratory method, like convergent beam electron diffraction (CBED).

We proceeded in a similar way as in $[14,15]$ : we followed the classical nucleation and growth theory [16], which predicts an Avrami time law for the evolution of the transformed (i.e. precipitated) volume fraction. By means of in-situ isothermal ageing, we have then extended the investigation to the successive growth and coarsening processes, attempting to investigate the character of the reaction as a function of time and temperature. Indirectly, the effect of the energy terms in equation (1) on the reaction could be observed. Neutron diffraction was particularly useful to determine the evolution of the volume fraction of the $\gamma$ ' phase, while synchrotron radiation allowed determining the lattice parameter of the two phases, thanks to the high resolution achievable.

Therefore, the present work describes the real-time evolution of both the volume fraction of the $\gamma$ and $\gamma^{\prime}$ phases and of their lattice mismatch at several temperatures, starting from the solutionised state (i.e. cooling from the solvus temperature $\mathrm{T}=1525 \mathrm{~K}$ ). It also discloses the stabilization and the relaxation of the coherency strains during long ageing treatments.

Finally, it opens the possibility for future in-situ investigations, as well as for industrial on-line process control.

\section{Experimental Details}

A high-temperature mirror furnace [17], see Fig.1, capable of reaching more than $2000 \mathrm{~K}$ with a temperature uniformity of $\pm 5 \mathrm{~K}$ within $5 \times 5 \times 5 \mathrm{~mm}^{3}$ at the sample position, was used for all in-situ investigations. It also allows the sample environment to be flowing inert gas or vacuum, in order to suppress oxidation as much as possible. Those options were not used, since for neutron and high-energy synchrotron radiation diffraction oxide layers play a minor role. In fact, working in air environment gives the advantage of easy optical inspection. Neutron diffraction and synchrotron radiation are particularly advantageous techniques to follow real-time transformations with a half-life of about 15 down to 1 minute time-scale, respectively. Their penetration into matter allows the use of relatively big samples. The samples basically need neither machining nor chemical etching. As already mentioned, they both completely disregard the presence of the oxide layer, which inevitably forms at high temperatures. Finally, the availability of high-temperature furnaces for neutron diffractometers is of capital importance for this kind of investigations. Those advantages render neutron and synchrotron radiation diffraction better suited to this kind of investigation, particularly with respect to laboratory techniques.

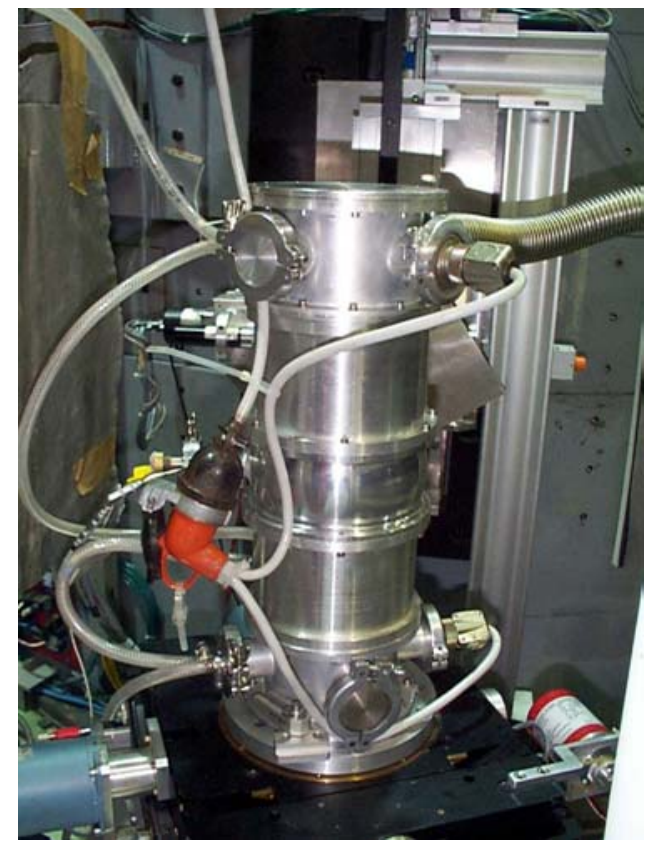

Fig.1 - The mirror furnace for high temperature in-situ investigations, developed at the ILL, used in Berlin (E3) and Hamburg (BW5). In both cases a lighter version, without the central aluminum ring, was used to facilitate optical inspection.

Neutron diffraction measurements were carried out at the BER II reactor of the HMI-Berlin, Germany, on the E3 diffractometer [18], equipped with a position sensitive detector (PSD). A wellcollimated primary beam (10' primary and secondary collimation, respectively upstream and downstream of the monochromator), was used with wavelength $\lambda=1.370 \AA$ and a beam size of $3 \times 5$ $\mathrm{mm}^{2}$ (the sample size was $5 \times 5 \times 8 \mathrm{~mm}^{3}$ ). The 200 and $400 \gamma+\gamma$ ' and $300 \gamma^{\prime}$ (superlattice) reflections were exploited, as the scattering properties of the alloying elements to neutrons (listed in Table 1) give different structure factors for the alloy mixture and the $\gamma^{\prime}$ $\mathrm{Ni}_{3}(\mathrm{Al}, \mathrm{Ti})$ stoichiometric compound [19]. The time resolution to acquire reasonable statistics was around 5-10 min. Measurements were done with the sample at the Bragg position in the $\omega$ and $\chi$ tilting angles for all the peaks.

Synchrotron radiation diffraction measurements were performed on the high-energy beamline BW5 of the HASYLAB facility at DESY, Hamburg, Germany [20]. On BW5, a 'three crystal' configuration was used, with Si-(220) perfect monochromator and analyser crystals. The wavelength used was $\lambda=0.1129 \AA$ (corresponding to an energy of $E=109.8 \mathrm{keV}$ ) and all measurements were performed in the so-called $(+,-,+)$ mode [21]. A beam size of $3 \times 3 \mathrm{~mm}^{2}$ was preferred to speed-up data acquisition. Nevertheless, to acquire peaks with sufficient resolution ( $2 \theta$ step of down to $2.5 \times 10^{-4} \circ$ ), a total counting time of about 5 minutes was required for each peak. 
Three other samples were investigated on the beamline PETRA II at HASYLAB, Hamburg, Germany [22], with a similar experimental arrangement, since the two beamlines share the same general layout. Those samples were pre-aged at $1270 \mathrm{~K}$ for $25 \mathrm{~h}$ and $100 \mathrm{~h}$ and at $1120 \mathrm{~K}$ for $150 \mathrm{~h}$ (and quenched to freeze their microstructure). All samples, with size $5 \times 5 \times 8 \mathrm{~mm}^{3}$, were cut from the same single-crystal ingot. They were previously oriented in a Laue Camera, with faces parallel to the $\{100\}$ lattice planes.

The two sets of measurements were somewhat complementary. On E3, in spite of the relatively low resolution, the scattered intensity proved to be enough to follow the phase kinetics. In order to keep reasonable counting times, a coarse $2 \theta$ angular step was used $\left(0.2^{\circ}\right)$. Measurements on BW5 were aimed at a better angular resolution. This is why the time resolution was slightly sacrificed for the angular one.

A stepwise temperature profile was adopted on E3, using reasonably long ageing times for each temperature value (see Fig.2). On BW5, a saw tooth temperature profile (Fig.2) was adopted. In this case, longer ageing times were used at $1270 \mathrm{~K}$ and $1120 \mathrm{~K}$. This allowed monitoring also the coarsening stage of the reaction at each ageing temperature, as the volume fraction saturates. The sample temperature was monitored by a Pt-PtRh thermocouple in constant thermal contact with the sample. A shallow hole of the same diameter of the thermocouple was drilled in the sample and the thermocouple was fitted into it. The Eurotherm controller allowed a fine temperature tuning (up to \pm 3 $\mathrm{K}$ ), in a very short time (about $2 \mathrm{~min}$ ), so that the bigger source of error in the temperature determination came from the inhomogeneity of the focusing region. The whole sample must be thought to undergo gradients up to $50 \mathrm{~K}$, so that all temperatures suffer from an uncertainty of up to $\pm 25 \mathrm{~K}$.

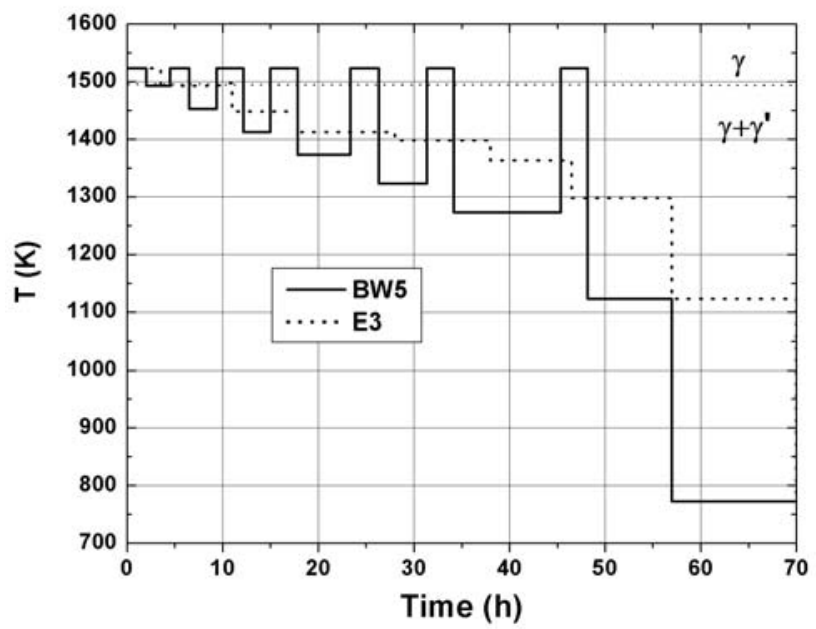

Fig.2- Typical schematic sample temperature profile during the real-time synchrotron radiation and neutron diffraction measurements on BW5 and E3. The pure $\gamma$ region is sketched, for sake of clarity, above $\mathrm{T}_{\mathrm{c}}=1475 \mathrm{~K}$, but $\gamma$ ' starts forming also around $1495 \mathrm{~K}$. Longer ageing times were chosen at 1280 $\mathrm{K}$ and $1120 \mathrm{~K}$ on BW5 (profile not shown).

\section{Results}

$\underline{\text { E3 }}$

The main parameter to calculate the volume fraction of the precipitate phase and its time evolution was the superlattice reflection peak area normalized to that of the nearest fundamental reflection [15,23]. This brings relative values, which are instrument independent. The integrated intensity was always calculated fitting each peak with a simple Gaussian function. This is reasonable for the superstructure reflections, which are expected to have a symmetric profile. The fundamental 200 and 400 reflections present a degree of asymmetry, which is proportional to the volume fraction of $\gamma^{\prime}$ and to the coherency strains (the lattice misfit $\delta$, see [24]). However, the resolution of the instrument allowed the detection of some profile asymmetry only to a small extent.

We considered the ratios of the 300 peak areas (PA) to the standard reflection 200, and we could draw the behavior of the integrated intensities as a function of time and temperature. This is represented in Fig.3. The whole profile shows, in the inset, the behavior of the volume fraction, calculated as below. As expected, it increases with the undercooling from the solutionising temperature $(1525 \mathrm{~K})$. If we assume, after the experimental results of $[3,10,24]$, that at $1100 \mathrm{~K}$ the volume fraction $\mathrm{f}$ lies around $40 \%$ (i.e. $f$ is essentially the same as at RT), then $f$ decreases as a function of temperature, as shown in the inset in Fig.3.

Although the element partitioning has not yet been thoroughly studied and is not known exactly at very high temperature, the $\gamma$ ' chemical composition will not vary significantly from that found at RT with atomic force microscopy (AFM) by Zhang et al. [25]. It can be then assumed that the scattering properties (see Table 1 and the discussion below) of the alloy do not vary when going from low to high temperatures. In addition, it was observed that time/temperature evolution of the 200 reflection shows a constant behavior, within the error bar. Finally, the measured integrated intensity of the fundamental reflection 200 is a factor of 9 to 10 larger than that of 300 , i.e. the ratio $\mathrm{I}_{200} / \mathrm{I}_{300} \sim 10$. This is in good agreement with the theoretical predictions. In fact, if $\mathrm{b}$ is the scattering length for thermal neutrons (Table 1), then $I_{300} \propto\left|F_{300}\right|^{2}$ $=\left|\mathrm{F}_{100}\right|^{2} \cong\left|\mathrm{b}_{\mathrm{Al}}-\mathrm{b}_{\mathrm{Ni}}\right|^{2}=0.476 \cdot 10^{-24} \mathrm{~m}^{2}$, and $\mathrm{I}_{200} \propto\left|\mathrm{F}_{200}\right|^{2} \cong \mid \mathrm{b}_{\mathrm{Al}}+$ $\left.3 \mathrm{~b}_{\mathrm{Ni}}\right|^{2} \cong|4<\mathrm{b}>|^{2} \cong 10.5 \cdot 10^{-24} \mathrm{~m}^{2}$. If we now take into account the geometrical factor $\sin 2 \theta$ and the Debye-Waller factors for the 200 and 300 reflections, we get $\mathrm{I}_{200} / \mathrm{I}_{300} \sim 12$ (above, $\langle\mathrm{b}\rangle$ is the average scattering length, defined as $\langle\mathrm{b}\rangle=\Sigma_{\mathrm{i}}\left(\mathrm{b}_{\mathrm{i}} \cdot \mathrm{f}_{\mathrm{i}}\right) / \Sigma_{\mathrm{i}}\left(\mathrm{f}_{\mathrm{i}}\right), \mathrm{f}_{\mathrm{i}}$ being the atomic percentage of the $\mathrm{i}$-th element, listed in Table 1).

BW5.

The typical order of magnitude of $\delta$ is about $10^{-3}$. Therefore, the separation of the $\gamma$ and $\gamma^{\prime}$ peaks would require a resolution of about $5 \times 10^{-4}$. This was impossible to achieve with neutron diffraction, also because of the much bigger beam angular divergence of neutron with respect to synchrotron radiation beams. On the other hand, on BW5 the resolution, measured with a perfect $\mathrm{Si}-(220)$ single crystal at sample position, easily reached $5 \times 10^{-5}$. This allowed to record not only the $\gamma$ and $\gamma$ ' peak positions, but also to see the further peak splitting due to the dendritic structure typical of these alloys. Particle of different sizes are present, depending on their location as shown in Fig.4. Correspondingly, the diffraction patterns show many peaks other than the main $\gamma$ and $\gamma^{\prime}$ (see [26] for a thorough discussion).

The necessity of using a fine step-scan to reveal all the details of the diffraction patterns, still limiting the counting times, obliged us to use only the fundamental reflection 200 at high temperatures. The superstructure reflection 100 was mapped at room temperature and on the 3 pre-aged samples mentioned above [26]. The results of the real-time measurements are shown in Fig.5 
and Fig.6. The intensity of the two main peaks follows a specular behaviour, as expected. While the $\gamma^{\prime}$ phase grows, the $\gamma$ phase volume fraction $f$ decreases. In Fig. 5 the total sum of the intensities was normalised to 1 .

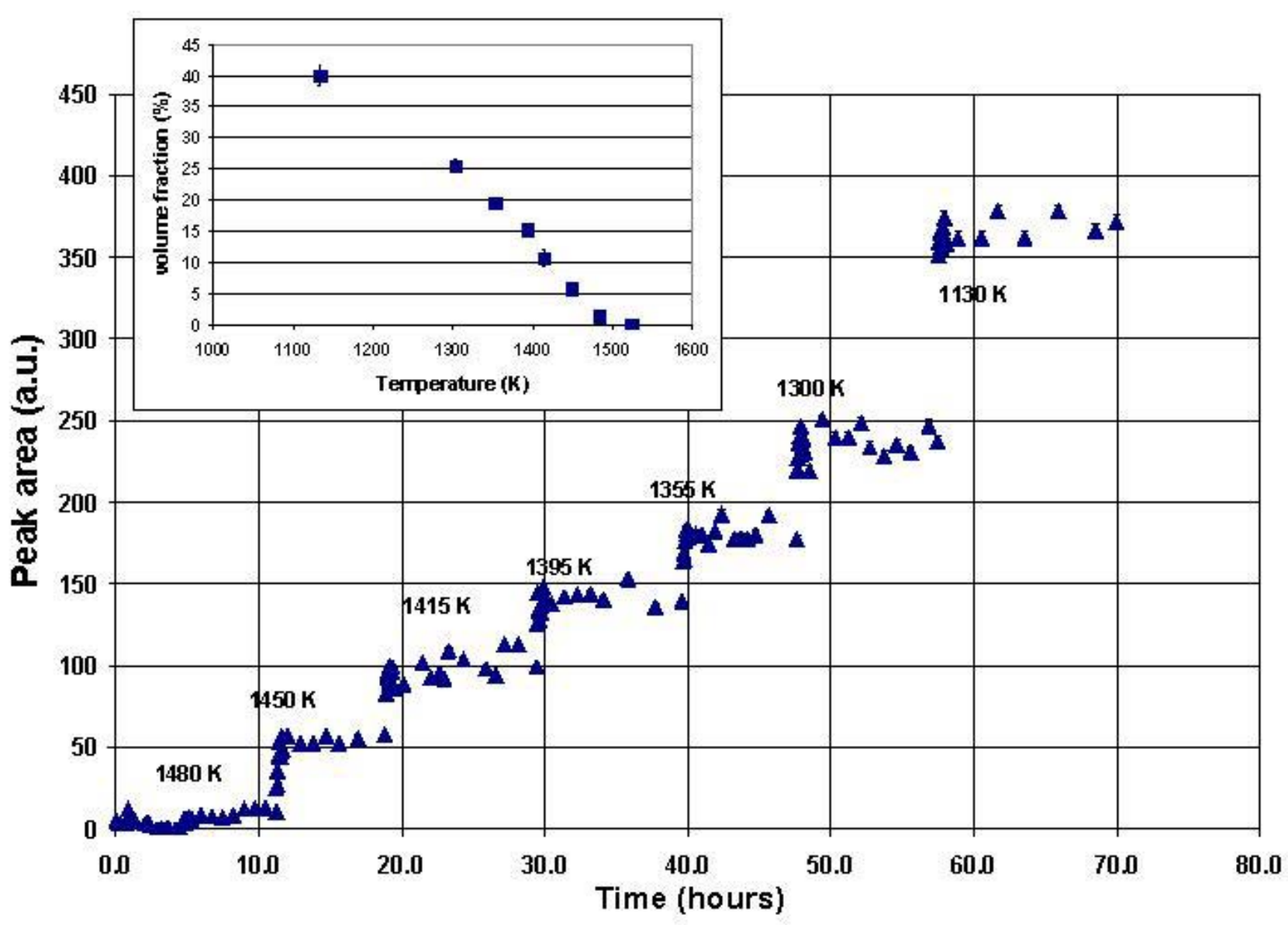

Fig.3- Normalised $\gamma^{\prime}$ peak area as a function of temperature and time. Measurements were done on E3, HMI, Berlin. In the inset the calculated volume fraction $f$ (its saturation value at each temperature) is shown. The calculation assumes that at $1100 \mathrm{~K} f=40 \%$

As mentioned above, only the main $\gamma$ and $\gamma^{\prime}$ peaks were taken into account in the intensity and lattice parameter calculations. The presence of the reported sub-peaks was basically neglected, because they have a very small integrated intensity and their origin is not clear. In fact, not only the $\gamma^{\prime}$-depleted matrix regions and interdendritic areas, but also the precipitation of TCP (topologically close packed) phases, mentioned in [27,28] can bring about additional diffraction contribution. The results of the lattice mismatch calculations, as defined in Eq.(2), are shown in Fig.6.

The behavior of the integrated intensity shown in Fig.5 is very similar to that of Fig.3. At $1120 \mathrm{~K}$ the $\gamma^{\prime}$ phase seems to have slightly more than the theoretical $40 \%$ volume fraction, but this is due to the presence of the extra $\gamma$ phase peaks, as mentioned above. On discarding them, one also discards the small amount of $\gamma$ volume fraction missing for an exact match with the theoretical balance.

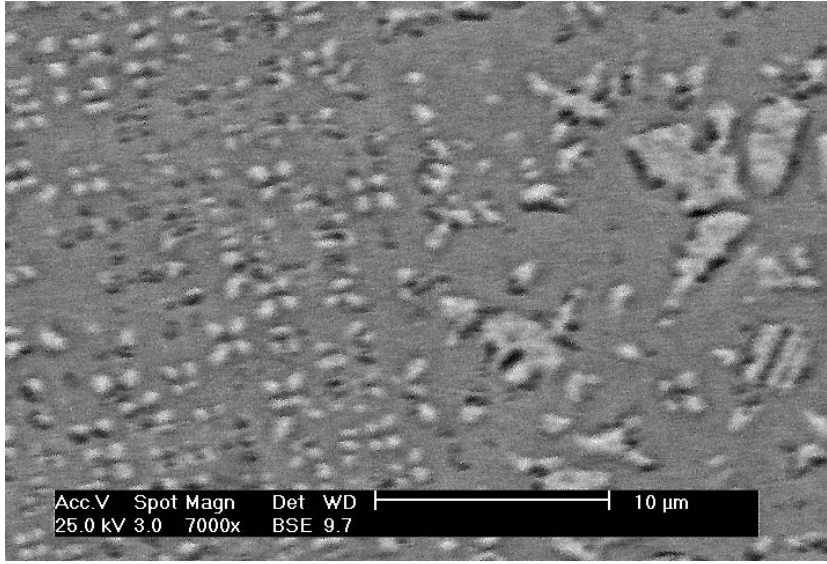

Fig.4- Microscopic consequences of the dendritic structure in the SC16. In the interdendritic zones the particles become bigger, their shape is irregular and they are not as ordered as in the dendritic zones. 
The behaviour of the misfit, as measured in-situ on BW5, (Fig.6) is decaying at every temperature, as the $\gamma$ ' integrated intensity. The behaviour of the misfit, as measured on PETRA II, Hasylab,
Hamburg, Germany, for the three pre-aged samples at 1270 and $1120 \mathrm{~K}$ is shown in Fig.7.

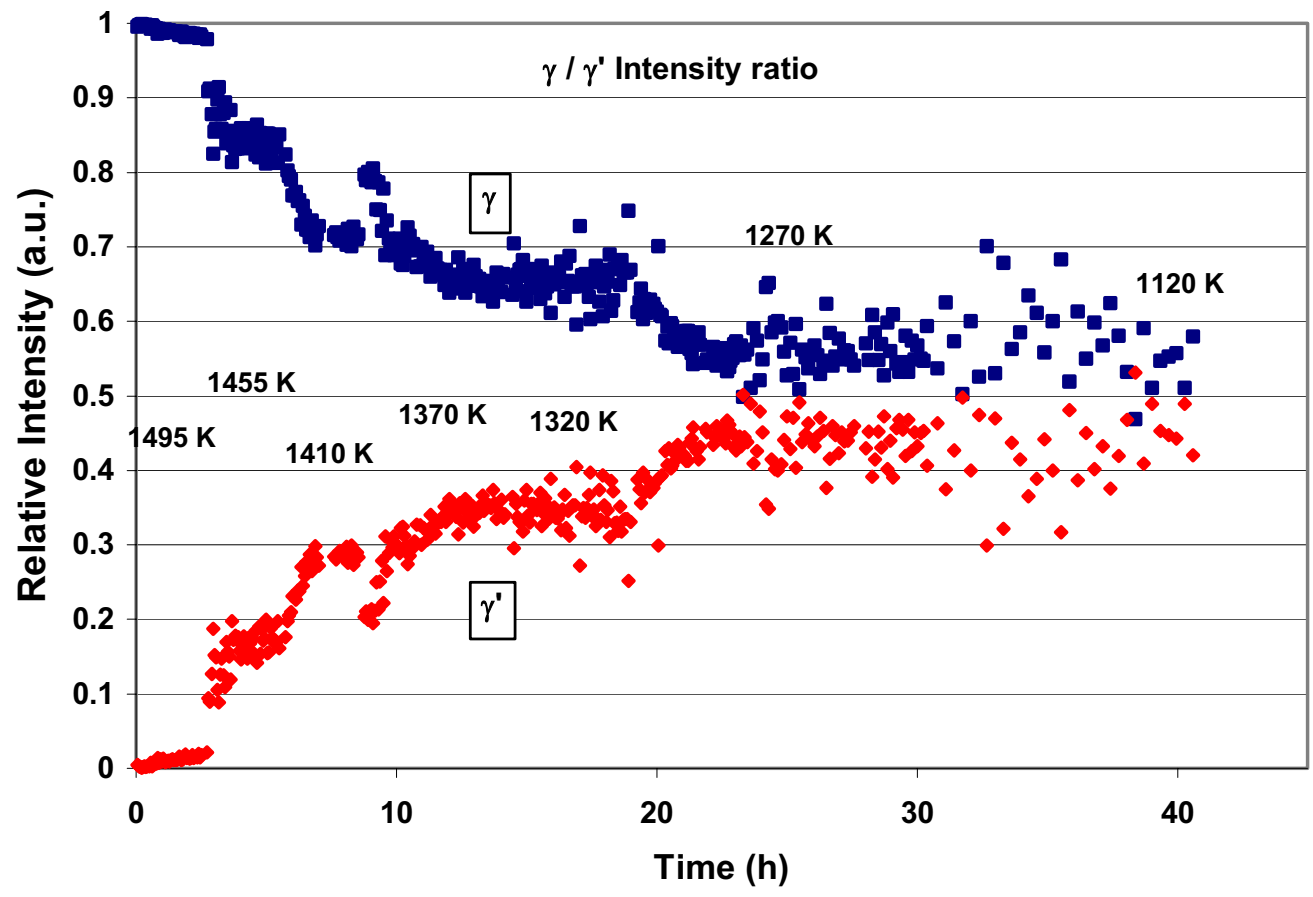

Fig.5- Integrated Intensity of the $\gamma$ and $\gamma^{\prime}$ main peaks as a function of time and temperature. The total intensity has been normalised to 1 .

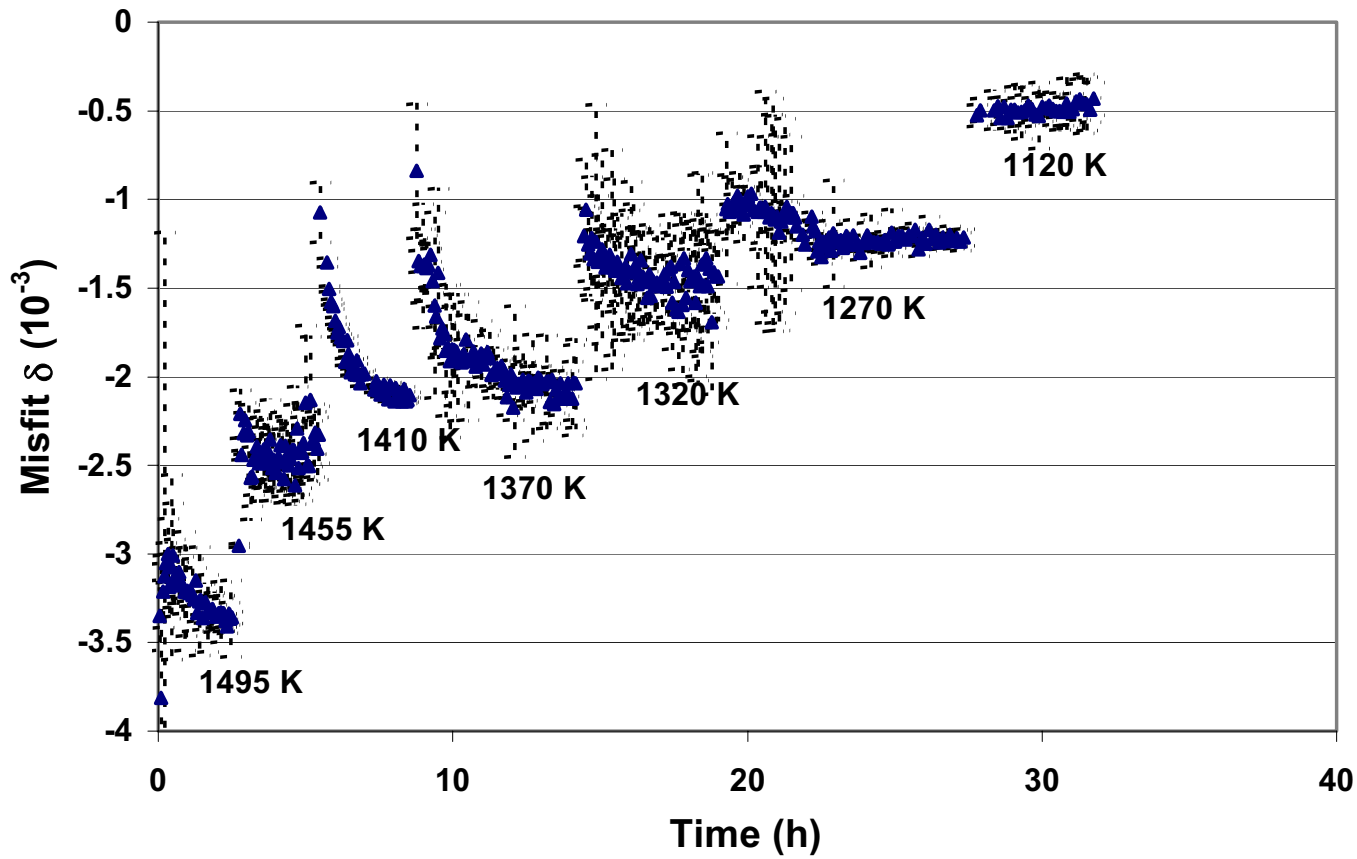

Fig.6- Lattice misfit as a function of time and temperature, as measured on BW5 at the HASYLAB synchrotron, Hamburg, Germany. 

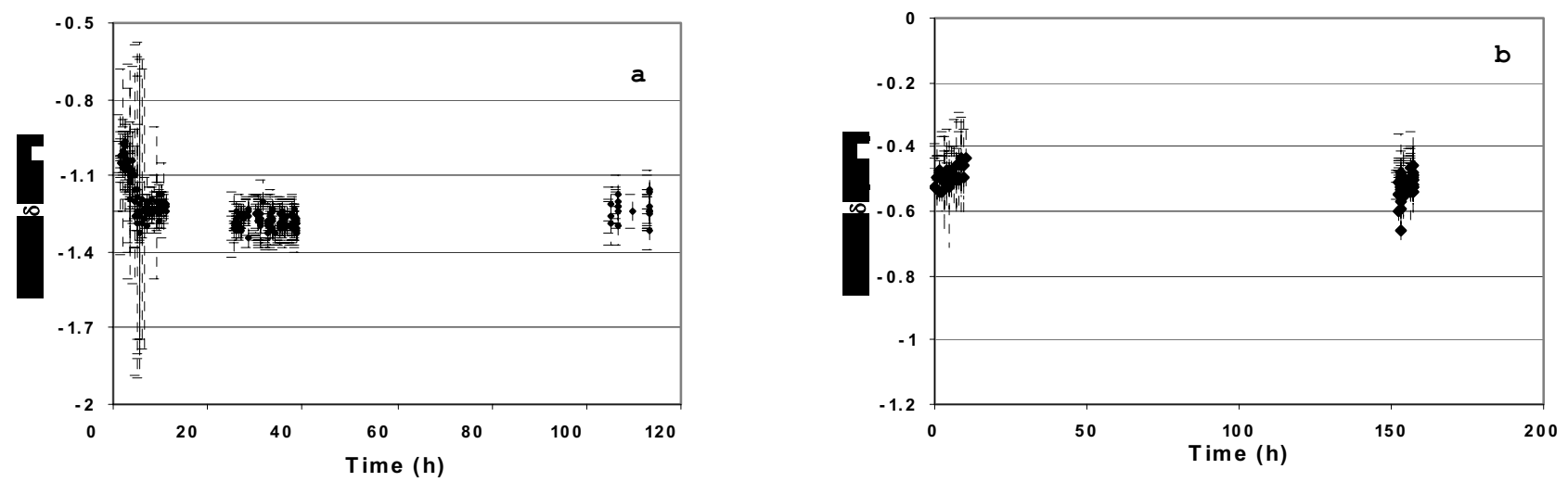

Fig.7- Lattice misfit as a function of time at $1270 \mathrm{~K}$ (a) and $1120 \mathrm{~K}$ (b). Three extra samples were prepared with pre-ageing of 25 , and $110 \mathrm{~h}$ at $1270 \mathrm{~K}$ and $150 \mathrm{~h}$ at $1120 \mathrm{~K}$.

\section{Discussion}

At each ageing temperature the time evolution of the integrated intensity ratio between superlattice and fundamental reflections or the volume fraction $f v s$. time $t$ satisfy a Johnson-Mehl-Avrami's law $[14,29,30]$, if we assume a classical kinetic theory.

The temporal behaviour can be written as

$$
f=f_{0}\left(1-e^{-c t^{n}}\right)
$$

where $c$ is a multiplying factor, $n$ is an exponent which defines the character of the reaction [31] and $f_{0}$ is the saturation level (in arbitrary units). An example is shown in Fig.8.a.

Results of the Avrami analysis are summarized in Table 2. We note that below $1480 \mathrm{~K}$, the exponent $n$ increases going from values $\leq 1.5$ to values $>1.5$ and then comes back to values $\leq 1.5$. Above $1480 \mathrm{~K}$ the exponent lays around 4. If we follow [31,32], when $n \leq 1.5(\geq 1.5)$ the nucleation rate is about constant (increases), namely its derivative is zero (positive) and the reaction is bulk (i.e. diffusion) controlled. If $n \sim 4$ then the reaction is strongly surface controlled [32] and the main contributions to the nucleation rate are the rate of transmission across the interface and the diffusional growth of very small objects [31,32]. In other words, the geometry of the particles (large surface-to-volume ratio) drives the reaction to be surfacecontrolled, but long-range diffusion is obviously always present. The relatively big error in the determination of $n$ is due to the strong coupling of the fitting variables in Eq.(3). The variation of the exponent seems to be genuinely due to the change of the reaction character, as the two sets of results corroborate each other.

The life-time for the reaction to saturate (at each ageing temperature) can be calculated as the time $\tau_{I}$ for the volume fraction $\mathrm{f}$ to reach $f_{0} / 2$

$$
\tau_{I}=\left(\frac{\ln 2}{c}\right)^{\frac{1}{n}}
$$

The reaction rate is the defined by $W=1 / \tau_{\mathrm{I}}$.

The behaviour of $\delta v s$. time $t$, at each temperature T, was fitted with a simple exponential time decay $\delta=\delta_{0} \cdot e^{-t / \tau_{\delta}}$
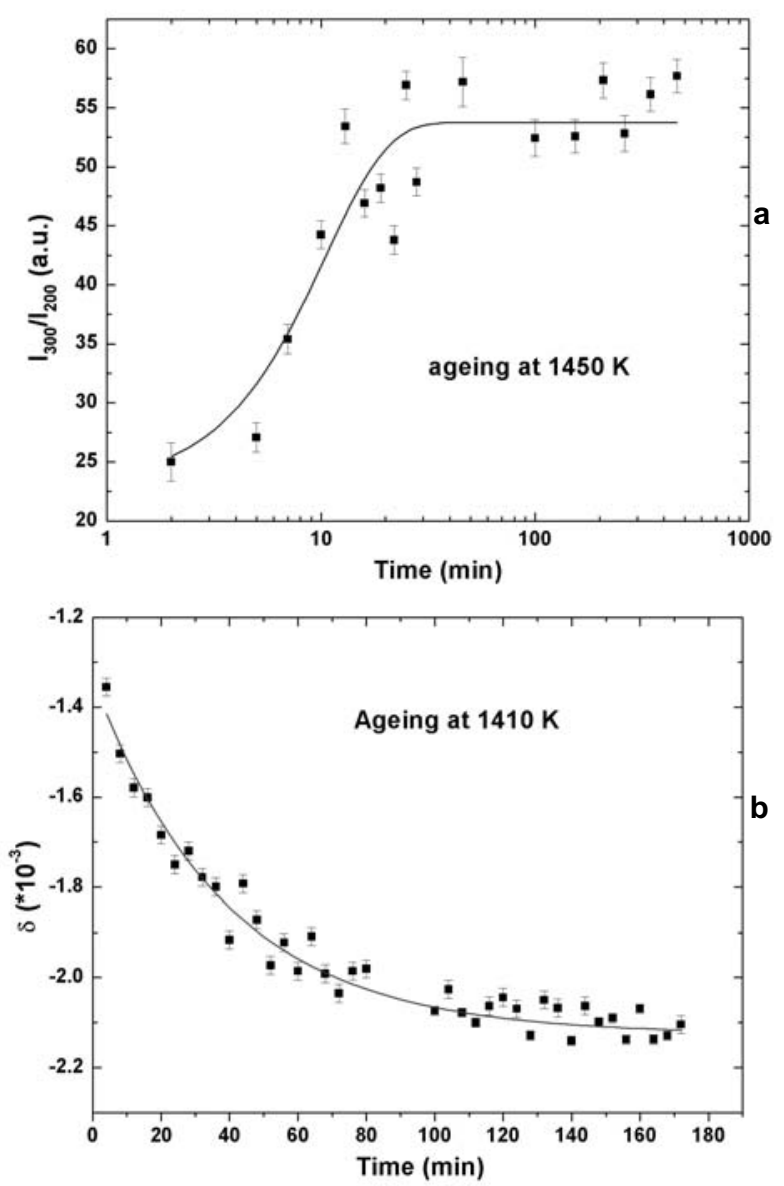

Fig.8- Examples of the growth of the integrated intensity (a) and of the time decay of the misfit (b) as a function of time, as measured on E3 (neutrons) and BW5 (synchrotron radiation), respectively. 
An example is given in Fig.8.b. We can also define the relaxation rate $W_{\delta}$ for the misfit as the inverse of the half-life $\tau_{\delta}$ displayed in Tab.3.

Table 2. Life-time $\tau_{I}$ and Avrami coefficients $n$ and $c$ for the total nucleation and growth reaction.

\begin{tabular}{|c|c|c|c|c|c|}
\hline $\mathrm{T}(\mathrm{K})$ & $\tau_{I}(\min )$ & $n$ & $\Delta n$ & $c\left(\mathrm{~min}^{-\mathrm{n}}\right)$ & $\Delta c\left(\mathrm{~min}^{-\mathrm{n}}\right)$ \\
\hline 1480 & 29 & 3.77 & 1.36 & $2.110^{-7}$ & $6.710^{-8}$ \\
1480 & 25 & 4.16 & 0.09 & $1.110^{-6}$ & $9.010^{-8}$ \\
1450 & 8 & 1.37 & 0.37 & 0.037 & 0.033 \\
1410 & 9 & 1.50 & 0.43 & 0.012 & 0.008 \\
1380 & 15 & 1.31 & 0.49 & 0.030 & 0.028 \\
1350 & 8 & 1.60 & 0.55 & 0.027 & 0.012 \\
1300 & 9 & 1.84 & 0.77 & 0.015 & 0.012 \\
1130 & 10 & 1.30 & 0.72 & 0.035 & 0.014 \\
\hline
\end{tabular}

Although at some temperatures a relatively large error was found, the behaviour of $\delta$ as a function of time can be clearly recognised and the fit was done weighting on the experimental errors. The half-life for relaxation is displayed in Table 3.

At each temperature, after cooling down from the solvus point, nucleation is expected to be the driving mechanism for the solidstate reaction in the first stages. We have seen above that nucleation is replaced slowly by growth and, after long times $(t>$ $4 \mathrm{~h})$, by coarsening. Therefore, for what the misfit is concerning, we can guess that, at high temperatures $(\mathrm{T}>1370 \mathrm{~K})$, particles are born coherent and their mismatch is initially very low (the lowest compatible with the difference in thermal expansion coefficients, CTEs, between the two phases). As the time goes on, the $\gamma^{\prime}$ particle size increases, and the misfit becomes larger (i.e. more negative). Below $1250 \mathrm{~K}$, the coarsening rate is very low and at $1100 \mathrm{~K}$ the misfit is basically constant from the very early stages (see Fig.6). This strongly supports the idea that newly nucleated particles drive the time evolution of $\delta$ and that the misfit depends essentially on the difference of CTE, as confirmed by neutron diffraction [33]. At each temperature, the asymptotic value of the misfit is reached once the $\gamma^{\prime}$ precipitates become fully incoherent to the $\gamma$ matrix. This has been checked with long-term ageing at $1370 \mathrm{~K}$ (for $25 \mathrm{~h}$ and $100 \mathrm{~h}$ ) and $1120 \mathrm{~K}$ (for $150 \mathrm{~h}$ ), see Fig.7. After the initial time decay, in both cases the misfit stays constant until $170 \mathrm{~h}$ ageing treatment. This proves that diffusion, which for example tends to smear the dendritic structure, plays an eminent role during nucleation only, and the element partitioning between $\gamma$ and $\gamma^{\prime}$ does not influence $\delta$, if it ever changes significantly.

In the parentheses of Tab. 3 the values obtained by neutron diffraction, as measured on the powder diffractometer E9, at the HMI, Berlin, Germany, are presented. The small discrepancies between the two sets of results can be explained by means of the different temperature profile adopted (on E9 a cascade ladder-like profile was used, like on E3).

We can now apply a simple model to the reaction rate $W$ and to the relaxation rate $W_{\delta}[4,32,34]$. The reaction rate $W$ increase with diffusion, that is with increasing temperature, approaching the solutionising limit $\mathrm{T}_{0}=1525 \mathrm{~K}$, as an energy activated process, which follows an Arrhenius behaviour

$$
W \sim A e^{-\frac{Q}{R T}}
$$

where $Q$ is in our case a sort of 'average' activation energy and $R$ the gas constant. However, in our case $Q$ has no particular meaning, since the reaction changes its character.

Table 3. Relaxation rate $W_{\delta}$ and half-life $\tau_{\delta}$ for the misfit $\delta$, as measured on BW5. Their errors are also reported. Values in parenthesis have been measured with neutron diffraction on E9, HMI, Berlin, Germany.

\begin{tabular}{|c|c|c|c|c|}
\hline $\mathrm{T}(\mathrm{K})$ & $\tau_{\delta}(\min )$ & $\Delta \tau(\min )$ & $W_{\delta}\left(\mathrm{min}^{-1}\right)$ & $\Delta W_{\delta}\left(\mathrm{min}^{-1}\right)$ \\
\hline 1495 & 80 & 6 & 0.0125 & 0.001 \\
1455 & $(45)$ & $(9)$ & $(0.023)$ & $(0.0045)$ \\
1410 & $39(12)$ & $1(8)$ & 0.0256 & 0.00016 \\
1370 & $71(30)$ & $7(10)$ & 0.0141 & 0.0011 \\
1320 & 55 & 14 & 0.0182 & 0.0046 \\
1270 & 144 & 27 & 0.0069 & 0.0042 \\
1120 & $\infty(\infty)$ & - & $0(0)$ & - \\
\hline
\end{tabular}

$W$ should also increase with the undercooling $\Delta \mathrm{T}=\mathrm{T}_{0}-\mathrm{T}$, simply because, in a quasi-static process, the volume fraction of the precipitate phase is proportional to $(\Delta \mathrm{T})^{\alpha}(\alpha \geq 1)$. A minimum undercooling $\left(\Delta \mathrm{T}_{\mathrm{S}}=\right.$ supercooling $)$ has been found in classical works $[35,14]$. This has a strong dependence of the alloy composition. This was confirmed in the present work, as no significant increase of the superlattice reflection integrated intensity has been observed above $1500 \mathrm{~K}$, within a reasonable time. We found that $\Delta \mathrm{T}_{\mathrm{S}} \sim 30 \mathrm{~K}$. Finally, the temperature dependence of $W$ can be written as

$$
W=W_{0}(\Delta T)^{\alpha} e^{-\frac{Q}{R T}}
$$

According to [4,36], the exponent $\alpha$ assumes the value of 2 when bulk processes occur, whereas $\alpha=3$ when surface controlled reactions play an eminent role. This is shown in Fig.9. Fitting the $W$ data obtained from Eq.(4) with the function (7), we note that, although both models $(\alpha=2$ and $\alpha=3)$ can be well applied, the best fitted nucleation exponent is $\alpha \cong 2.7$ (the residuals are smaller). This would imply a mainly (but not completely) surface driven nucleation and growth reaction. These findings reflect what the Avrami exponents suggest (Table 2), confirming the statements above. Thus, it can be concluded that the Avrami analysis has been carried out self-consistently and the meaning of 'mainly surface reaction' must be taken, loosely, as driven by surface energy in competition with bulk diffusion processes. In other words the particles in the early stages of the reaction have still too a large surface/volume ratio for the nucleation and growth to be treated as bulk phenomena. The temperature where $W$ reaches its maximum $\left(\mathrm{T}_{\max }\right)$ was found to lay around $1320 \mathrm{~K}$.

The misfit relaxation rate $W_{\delta}$ also follows a pseudo-Arrhenius law (7), but for different reasons: $W_{\delta}$ decreases with the amount of diffusion and decreases with the undercooling. Therefore a law such as Eq.(7) can be used, with $\alpha=1$. This analysis is shown in Fig.10. The behavior of $W_{\delta} v s$. T has a maximum around $1420 \mathrm{~K}$ (Table 3). 


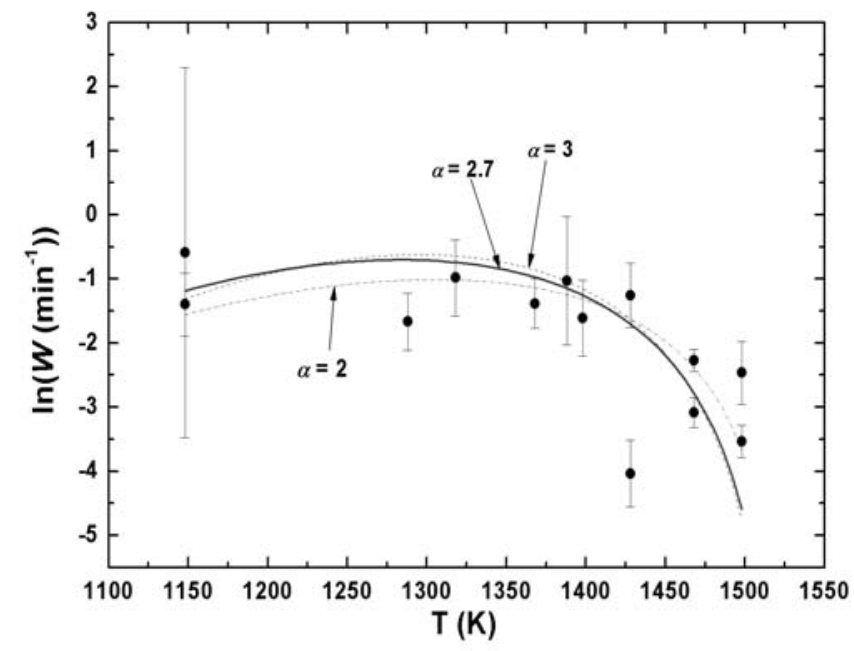

Fig.9- Pseudo-Arrhenius plot of the reaction rate $W$ as a function of temperature. Different exponents $\alpha$ are shown.

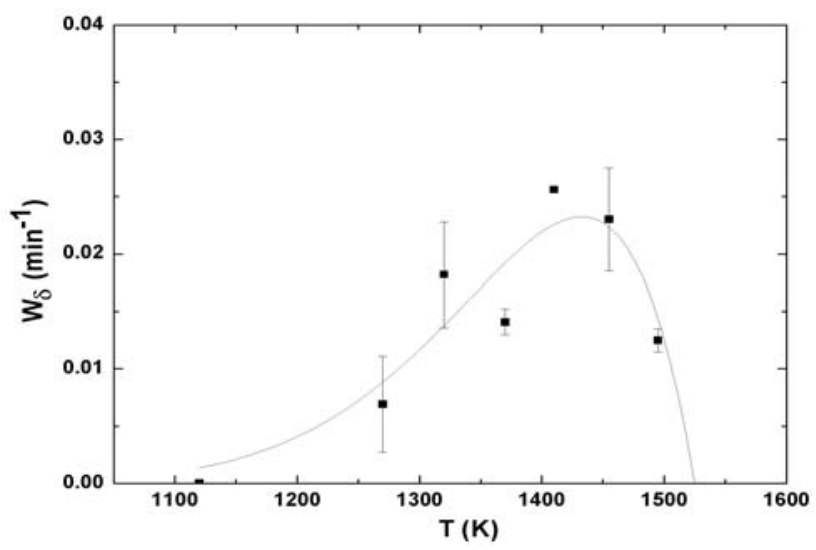

Fig.10- Pseudo-Arrhenius (Eq.(7)) plot of the relaxation rate $W_{\delta}$, with $\alpha=1$.

The difference between the two maxima can be explained with the different nature of the phenomena under examination. The misfit $\delta$ influences and represents essentially the $E_{\delta}$ (strain energy) contribution to the energy balance, while the Integrated Intensity comprises strain, interface and diffusion energies. At high temperatures, diffusion is so strong and the particle size is so small, that the strain relaxation is slow. At intermediate temperatures the misfit relaxes more rapidly towards its asymptotic value, which implies that diffusion is overcome by the elastic energy. At relatively low temperatures there is little diffusion and the particles are already big and stable (the nucleation rate is smaller), so that the strains doesn't relax at all.

The results on the total reaction rate $W$ are in agreement with expectations of kinetic theory: at the beginning, the reaction is surface controlled, because the particle surface-to-volume ratio is very large. Then, bulk diffusion phenomena take over, as the size of the particles becomes more important. It can be reckoned that at the beginning the strain energy is very low because even if the misfit $\delta$ is high, because the particle volume fraction is very small. Therefore the nucleation energy $\Delta F_{\gamma}$, has to compete only with $\sigma_{\gamma \gamma}$. At lower temperatures, the elastic term $E_{\delta}$ in Eq.(1) gets more importance and the reaction proceeds by conventional nucleation and growth.

Finally we note that at each temperature, the misfit (and indirectly the coherency strains) relaxes after $2-4 \mathrm{~h}$ and reaches its asymptotic value. If coherency would be lost only in longer times, the value of $\delta$ would change also during long-term ageing, whereas we found it constant. This is confirmed by SEM images (not reported here, see [38]), which show $\gamma^{\prime}$ dendrites, typical of a structure without coherency at interface.

The asymptotic values of the misfit at each temperature are displayed in Fig.11. Here, the data obtained in [37] are also shown, as collected heating a SHT sample from RT up to $1170 \mathrm{~K}$. A kind of hysteresis can be seen, and values of $\delta$ during cooling are always smaller. However, it must be noted that:

- The temperature where $\delta$ approaches 0 seems to be the same in both cases, i.e. about $770 \mathrm{~K}$

- The microstructure of the alloy after SHT is much more "regular" than that during any of the ageing treatments used in the cooling process (i.e. the arrangement is periodic and the shape is smoothly cuboidal rather than polyhedral).

Therefore, we can assume that $E_{\delta}(\mathrm{T})$ is the same function regardless of the process (depending only on the balance between shape and misfit effects), while $\delta(\mathrm{T})$ is also a function of the process underway, i.e. of the microstructure. If we combine all the results shown in this work, we are able to draw some interesting conclusions. In [37] it was found that the lattice misfit of a SHT (or of an as-cast) sample does depend mainly on the difference in the thermal expansion coefficient (CTE) of the two phases. The present results confirm and extend this finding: The hysteresis shown in Fig.11 can be explained if we suppose $E_{\delta}(\mathrm{T})$ being the same function of $\mathrm{T}$ during both cooling and heating, while the shape and the size (and their distributions) of the particles are different according to the process. In such a way $\delta$ can then get different values at the same temperature, if measured during heating or cooling, although the CTE difference must not depend on the process (heating or cooling).

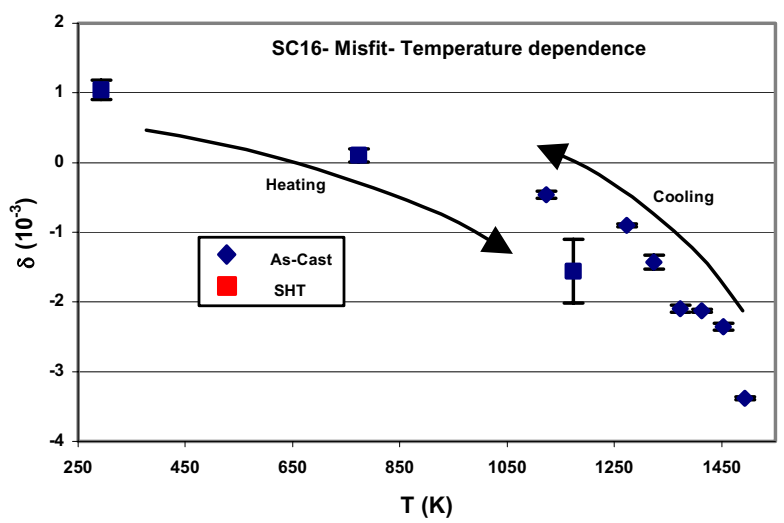

Fig.11- Behavior of the misfit during cooling and heating (see [37]). The misfit is positive at room temperature and goes to zero at about $770 \mathrm{~K}$. 


\section{Conclusions}

In the present work the authors tried to cast a new light on the kinetics of technical (i.e. non-ideal) Nickel-base superalloys:

- A 'real time' study of the $\gamma$ ' volume fraction, using in-situ neutron diffraction was performed in order to follow first the dissolution and then the early stages of the $\gamma$ ' particle nucleation and growth as well as the successive isothermal coarsening. The investigation started directly from the solvus point $(1525 \mathrm{~K})$. This procedure follows closely the heat treatment route of industrial components

- According to classical kinetic theory, an Avrami-like time dependence of the normalized integrated intensity of the superlattice reflections was used to model the varying $\gamma$ ' volume fraction $f$ at each temperature as a function of time. A stepwise temperature profile was used.

- It was found that the Avrami exponents change from values around 4 down to values around 1.5 and then grow up again to 1.8. Thus, a simple Arrhenius behavior cannot describe the early stages of the $\gamma$ ' precipitation and growth in this Nickelbase superalloy. Starting from simple classical kinetics considerations, a modified (pseudo) Arrhenius model was successfully used to fit the temperature dependence of the reaction rate. Although this model is adopted to describe discontinuous eutectoid reactions like bainite growth in steels [32], i.e. in a completely different context, its empiric use here allows describing competing bulk and surface effects.

- An increase of the reaction rate $W$ with undercooling (decreasing temperature) appeared, followed by a decrease below $\sim 1300 \mathrm{~K}$. The $W v$ s. $t$ curve seems to be well described by a nucleation term $(\Delta \mathrm{T})^{\alpha}$, with $\alpha \cong 2.7$ for surface driven discontinuous reaction with bulk reaction influences, and an 'average' growth Arrhenius term.

- Also the real-time variation of the $\gamma-\gamma$ ' lattice misfit was followed in an analogous manner, at several ageing temperatures, using synchrotron radiation diffraction. A relaxation from coherent state to an incoherent and probably unstrained one was observed at each temperature. The misfit goes from a relatively low value (near $-1 \times 10^{-3}$ for most temperatures) to an asymptotic value, which decreases as the sample is cooled down.

- Longer ageing times did not change the value of the misfit, thus suggesting that: a) diffusional composition changes do not affect the value of $\delta$ as a function of time, b) the reported loss of coherence takes place within $3 \mathrm{~h}$ time at each temperature. Point a) is particularly important if we consider that the microstructure changes: the $\gamma^{\prime}$ particles coarsen and the dendrites fade slowly. Point $b$ ) underlines that the misfit stays constant while particles are relaxing their strain, owing to the loss of coherency.

- The relaxation rate has its maximum around $1420 \mathrm{~K}$. This means that the strain energy will play an eminent role, at this temperature, during the early stages of nucleation.

- A hysteresis has been found in the heating/cooling cycle, as retrieved from present and previous data. This is explained assuming that $E_{\delta}(\mathrm{T})$ is the quantity strictly depending on the $\gamma-$ $\gamma$ ' CTE mismatch, not $\delta$ itself.

\section{Acknowledgements}

Financial support (contract Re 688/35-1,2) from the Deutsche Forschungsgemeinschaft (DFG) is acknowledged.
Hermann Franz and Martin von Zimmermann, HASYLAB, Hamburg, Germany provided experimental help on the BW5 and Petra II beamlines and data interpretation support. Walter Reimers (Technical University, Berlin, Germany), Philip Withers (Manchester Materials Science Centre, Manchester, UK) and Gaspar Gonzalez-Doncel (CENIM, Madrid, Spain) provided many hints and useful discussions.

\section{References}

[1] ASM Standards of Metals, Metals Park, Ohio, 1993.

[2] N.S.Stoloff: Int.Mat.Rev. 34 (1989) 153-161.

[3] T.Malow, J.Zhu, R.P.Wahi: Z.Metallk.de 85 (1994) 9-18.

[4] P.Haasen: 'Physical Metallurgy', Cambridge University Press, Cambridge, 1993.

[5] A.Fredholm, J.L.Strudel: Proc.of the $5^{\text {th }}$ Int. Symp. on Superalloys, TMS AIME, 1984, 211-220

[6] A.Baldan: Z.Metallk.de 83 (1992) 324-330

[7] A.A.Hopgood, J.W.Martin: Mater.Sci. Tech. 2 (1986) 543546.

[8] H.T.Kim, S.S.Chun, X.X.Yao, Y.Fang, J.Choi: J.Mat.Sci. 32 (1997) 4917-4923

[9] T.Malow: Diplomarbeit, TU Berlin, 1993.

[10] A.Royer, P.Bastie, M.Veron: Acta mater. 46 (1998) 53575368 .

[11] A.Royer, P.Bastie : Mater.Sci.Eng. A234-236 (1997) 11101113.

[12] H.A.Kuhn, H.Biermann, T.Ungar, H.Mughrabi : Acta metall. mater. 11 (1991) 2783-2794.

[13] H.Wendt, P.Haasen: Acta metall. 31 (1983), 1649.

[14] I.S.Servi, D.Turnbull: Acta metall. 14 (1966) 161-169.

[15] J.R.Stewart, R.Cywinski: J.Phys.:Condens.Matter 11 (1999) 7095-7102.

[16] D.Turnbull, Phase Changes, Solid State Physics 3 (1956) 226

[17] G.Lorenz, R.B.Neder, J.Marxbreiter, F.Frey, J.Schneider: J.Appl.Cryst. 26 (1993) 632.

[18]

http://www.hmi.de/bensc/instrumentation/instrumente/e3/e3.html. [19] B.D.Cullity: Elements of X-Ray Diffraction- Addison and Wesley, Reading (MA), 1978.

[20] R.Bouchard, D.Hupfeld, T.Lippmann, J.Neuefeind, HB.Neumann, H.F.Poulsen, U.Rütt, T.Schmidt, J.R.Schneider, J.Süssenbach, M.von Zimmermann: J.Synchr. Rad. 5 (1998) 90

[21] U.Rütt, H.B.Neumann, H.F.Poulsen, J.R.Schneider: J.Appl.Cryst. 28 (1995) 729

[22] R.Kampmann, T.Lippmann, J.Burmester, J.F.dos Santos, H.Franz, M.Haese-Seiller, M.Marmotti: Nucl. Inst. Meth. A 467468 (2001) 1261-1264

[23] O’Hare, J.S.O. Evans, R. Francis, S. Price, S. O’Brien: Mat. Sci. Forum 278-281 (1998) 367-378.

[24] A.Müller : Ph.D.Thesis, TU Berlin, 1995.

[25] Y.Zhang, N.Wanderka, G.Schumacher, R.Schneider, W.Neumann: Acta mater. 48 (2000) 2787-2793.

[26] H.Pinto, G.Bruno: J.Synchr.Rad. . 10 (2003) 148-153

[27] R.Gilles, D.Mukherji, P.Strunz, A.Wiedenmann, R.P.Wahi (1997). Z. Metallkde 88, 518

[28] M.Durand -Charre. The microstructure of superalloys, Amsterdam: Gordon and Breach Science Publishers, Glasgow, 1997

[29] D.H.Kirkwood: Acta metall. 18 (1970) 563-570.

[30] M.Avrami: J.Chem.Phys. 7 (1939) 1103.

[31] J.D.Livingston, J.W. Cahn: Acta metall. 22 (1974) 495. 
[32] R.D.Doherty: Chapter 14, in: R.W.CAHN, P.HAASEN, 'Physical Metallurgy', North-Holland, Amsterdam, 1983.

[33] G.Bruno, H.Cavalcanti Pinto : Mat. Sci. Techn. 19 (2003) 567-572.

[34] D.R.Askeland: 'The science and engineering of materials', Chapman and Hall, London, 1990.
[35] M.P.Jackson, M.J.Starink, R.C.Reed: Mat.Sci.Eng. A264 (1999) 26-38

[36] J.W.Cahn: Acta metall. 7 (1959) 18.

[37] G.Bruno, G.Schumacher, H.Cavalcanti Pinto, C.Schulze : Met.Trans.34A (2003) 193-197

[38] G.Bruno, H.Cavalcanti Pinto, P.Strunz, M.Wilhelm, in preparation (2004) 